City University of New York (CUNY) CUNY Academic Works

1991

\title{
Relativistic Wave Equation For Anyons
}

R. Jackiw

Massachusetts Institute of Technology

V. Parameswaran Nair

City College of New York

\section{How does access to this work benefit you? Let us know!}

More information about this work at: https://academicworks.cuny.edu/cc_pubs/385

Discover additional works at: https://academicworks.cuny.edu

This work is made publicly available by the City University of New York (CUNY).

Contact: AcademicWorks@cuny.edu 


\title{
Relativistic wave equation for anyons
}

\author{
R. Jackiw \\ Center for Theoretical Physics, Laboratory for Nuclear Science \\ and Department of Physics, Massachusetts Institute of Technology, Cambridge, Massachusetts 02139 \\ V. P. Nair \\ Physics Department, Columbia University, 538 West 120th Street, New York, New York 10027
}

(Received 3 December 1990)

\begin{abstract}
Construction of one-particle states as unitary representations of the Poincare algebra in $2+1$ dimensions shows that an anyon has one polarization state. However, for nonzero spin manifestly linear and covariant realizations of Lorentz transformations require more than one field component, and an infinite number is needed when the value of spin is not an integer or half-integer. We discuss the relation between these two aspects of Poincare symmetry. In particular, we construct a relativistic equation for anyons where the number of physical polarizations is reduced to one by virtue of a gauge symmetry or equivalent constraint.
\end{abstract}

\section{INTRODUCTION}

Theories of anyons or particles with arbitrary spin and statistics in $2+1$ dimensions have been attracting considerable attention, partially motivated by potential applications to the fractional quantum Hall effect, to high- $T_{c}$ superconductivity, and to the description of physical processes in the presence of cosmic strings. Since the original kinematical analyses revealed the possibility of anyons, ${ }^{1}$ many models have been proposed that realize anyonic states in a field-theoretic way. The solitons of the $\mathrm{O}(3) \sigma$ model with a Hopf term are particles of arbitrary spin and statistics. ${ }^{2}$ But the model has many other states of integral spin and therefore the $\mathrm{O}(3) \sigma$ model does not give a minimal theory of anyons. Another approach is to couple minimally point particles to a $\mathrm{U}(1)$ gauge field, sometimes called a statistical gauge field, whose dynamics is governed by the Chern-Simons action. ${ }^{3}$ The elimination of the gauge field leads to a redefinition of one-particle states; the new states carry arbitrary spin, determined by the coupling strength. In the relativistic context, possibly relevant to cosmic-string applications, carrying out the above procedure in a field theory (e.g., scalar or spinor fields coupled to a ChernSimons gauge field) does not produce a description for a free single particle with arbitrary spin. Indeed, it is not clear whether the only effect of the gauge field is to endow the particle with arbitrary spin or whether residual interactions are present. ${ }^{4}$ It is therefore of interest to construct a field theory where the fundamental fields are expected to represent the creation and annihilation of anyons.

As in any field theory, there are two aspects to the realization of Poincaré symmetry for anyons. First, the oneparticle states should carry a unitary representation of the Poincaré group; this can be achieved by use of an induced representation. Second, for a manifestly covariant theory the basic fields transform as a linear representation of the Lorentz group. The field equations and subsi- diary conditions (if any) then must be chosen so as to recover the one-particle Poincare group representation. ${ }^{5}$

In this paper we propose a field equation that attains this goal; just as the Dirac equation is appropriate for spin- $\frac{1}{2}$ particles and yields solutions that give a spin- $\frac{1}{2}$ representation of the Poincaré group, we offer an anyon equation that does the same job for planar particles with arbitrary spin.

In Sec. II we review the appropriate single-particle representation for the Poincaré group in $(2+1)$-dimensional space-time. In Sec. III we examine explicitly how solutions to familiar equations for spin $\frac{1}{2}$ and 1 give rise to these representations, and we observe that both equations can be presented in terms of the Pauli-Lubanski scalar. The remainder of the paper is devoted to our proposal. We describe and solve the field equations in Sec. IV. Section $\mathrm{V}$ offers a gauge-theoretic formulation of the subsidiary conditions that are used in Sec. IV. A Lagrangian for our field equation is given in Sec. VI, while concluding remarks comprise the final Sec. VII.

\section{POINCARÉ GROUP REPRESENTATION}

The representation of the Poincare group appropriate to one-particle states can be specified by values assigned to the invariants of the Poincaré algebra:

$$
\begin{aligned}
& {\left[J^{a}, J^{b}\right]=i \epsilon^{a b c} J_{c},} \\
& {\left[J^{a}, P^{b}\right]=i \epsilon^{a b c} P_{c},} \\
& {\left[P^{a}, P^{b}\right]=0,} \\
& \text { metric } g_{a b}=\operatorname{diag}(1,-1,-1) .
\end{aligned}
$$

Here $P^{a}$ and $J^{a}$ are translation and Lorentz-rotation generators: $P^{a}=(H, \mathbf{P}), J^{a}=\left(-M, \epsilon^{i j} L^{j}\right)$, with $M$ generating spatial rotations and $\mathbf{L}$ generating boosts. The constants $\hbar$ and $c$ are set to unity. In $2+1$ dimensions, the invariants are $P^{2}$ and $P \cdot J$ and the natural choice for them is ${ }^{5}$ 


$$
\left(P^{2}-m^{2}\right) \Psi=0, \quad(P \cdot J+s m) \Psi=0 .
$$

The first requirement is obviously the mass-shell condition and the second, involving the Pauli-Lubanski scalar $P \cdot J$, specifies the helicity, with $s$ the (arbitrary) value of spin that we can assign to the particle.

The solution to these equations is easily constructed. We take plane waves $\Psi(x)=\Psi(p) e^{-i p \cdot x}$ where $p^{a}$ are the momenta. The above equations apply to $\Psi(p)$ with $P_{a}$ realized as $i \partial / \partial x^{a}$ and replaced by the eigenvalue $p_{a}$. Since $p^{a}$ is subject to the mass-shell condition, $\Psi(p)$ depends only on p. $\Psi(p)$ carries a unitary representation for the little group of the rest-frame momentum vector $\hat{p}^{a}=(m, 0,0)$, viz., the two-dimensional rotation group:

$$
U(R) \Psi(p)=D(R) \Psi\left(R^{-1} p\right) .
$$

$U(R)$ is the unitary operator implementing the rotation on $\Psi$ and $D(R)$ comprise the spin-s representation matrices for rotations. Because planar rotations form the Abelian $U(1)$ group, $\Psi$ in our case is always a onecomponent object and $D(R)$ is just the phase $e^{-i s \omega}$, where $\omega$ is the angle of rotation. The action of the unitary operator $U(\Lambda)$, representing a general Lorentz transformation $\Lambda$ on $\Psi$, can then be constructed in the following manner. Let $B(p)$ be a Lorentzian transformation that boosts $p^{a}$ from its rest frame:

$$
B(p) \hat{p}=p .
$$

We then have

$$
U(\Lambda) \Psi(p)=D\left(R_{\Lambda, p}\right) \Psi\left(\Lambda^{-1} p\right),
$$

where the (Wigner) rotation $R_{\Lambda, p}$, depending both on $\Lambda$ and $p$, is defined as

$$
R_{\Lambda, p}=B^{-1}\left(\Lambda^{-1} p\right) \Lambda^{-1} B(p)
$$

and its one-dimensional representative $D\left(R_{\Lambda, p}\right)$ is the phase $e^{-i s \omega_{\Lambda, p}}$, involving the Wigner rotation angle $\omega_{\Lambda, p}$, which is determined by $p^{a}$ and the parameters $\omega^{a}$ of the Lorentz rotation $\Lambda$. To find $\omega_{\Lambda, p}$ note that the boosting transformation $B(p)$ is given by

$$
B(p)=e^{i \Omega_{a}(p) j^{a}},
$$

where $j^{a}$ represents the Lie algebra (2.1a) on vectors,

$$
\left(j^{b}\right)_{a c}=i \epsilon_{a}{ }^{b} c
$$

and

$$
\begin{aligned}
& \Omega_{a}(p)=\epsilon_{a b c} \frac{p^{b}}{|\mathbf{p}|} \eta^{c} \ln \frac{E+|\mathbf{p}|}{m}, \\
& E=p^{0}=\sqrt{|\mathbf{p}|^{2}+m^{2}},
\end{aligned}
$$

with $\eta^{a}$ defining the rest frame: $\eta^{a}=(1,0,0)$. Thus

$$
\begin{aligned}
{[B(p)]_{b}^{a} } & =\left[B^{-1}(p)\right]_{b}{ }^{a} \\
& =\delta_{b}^{a}-\frac{\left(p^{a}+\eta^{a} m\right)\left(p_{b}+\eta_{b} m\right)}{m(p \cdot \eta+m)}+\frac{2 p^{a} \eta_{b}}{m} .
\end{aligned}
$$

When the Lorentz transformation is parametrized by

$$
\begin{aligned}
& \Lambda=e^{i \omega_{a} j^{a}}, \\
& R_{\Lambda, p} \text { is } \\
& \quad R_{\Lambda, p}=e^{-i \Omega_{a}\left(\Lambda^{-1} p\right) j^{a}} e^{-i \omega_{b} j^{b}} e^{i \Omega_{c}(p) j^{c}},
\end{aligned}
$$

which explicitly reads

$$
\begin{aligned}
\left(R_{\Lambda, p}\right)_{b}^{a}= & \Lambda_{b}{ }^{a}+\Delta^{a}\left(\Lambda^{-1} p\right)\left(\eta_{b}-\Lambda_{b}{ }^{c} \eta_{c}\right) \\
& +\left(\eta^{a}-\eta^{c} \Lambda_{c}{ }^{a}\right) \Delta_{b}(p) \\
& +\left(\eta^{c} \Lambda_{c}{ }^{d} \eta_{d}-1\right) \Delta^{a}\left(\Lambda^{-1} p\right) \Delta_{b}(p),
\end{aligned}
$$

where

$$
\Delta^{a}(p) \equiv \frac{p^{a}+\eta^{a} m}{p \cdot \eta+m} .
$$

One readily verifies that $R_{\Lambda, p}$ is indeed a spatial rotation; for infinitesimal Lorentz transformations (2.13) reduces to

$$
\left(R_{\Lambda, p}\right)_{b}^{a}=\delta_{b}^{a}-i(j \cdot \eta)_{b}^{a} \omega \cdot \Delta(p)+\cdots,
$$

and this identifies the Wigner rotation angle, for an infinitesimal Lorentz transformation, as

$$
\omega_{\Lambda, p}=\omega \cdot \Delta(p)+\cdots .
$$

For later use let us remark that while the above development is carried out in the three-dimensional vector representation of the Lorentz group, formulas (2.6), (2.7), (2.11), (2.12), and (2.15) hold in arbitrary representations with the generator $j^{a}$ appropriately taken in that representation. Also, since infinitesimally

$$
\left(\Lambda^{-1} p\right)^{a}=p^{a}+e^{a b c} \omega_{b} p_{c},
$$

it follows from the definition (2.6) and the result (2.15) that the boost $B(p)$ in any representation satisfies

$$
B^{-1}(p)\left(-i \epsilon^{a b c} p_{b} \frac{\partial}{\partial p^{c}}+j^{a}\right) B(p)=j \cdot \eta \Delta^{a} .
$$

Because rotations acting on a state as in (2.3) give rise to the one-cocycle $e^{-i s \omega}$, the angular momentum generator $M=-J^{0}$ [with $U(R)=e^{-i \omega M}$ ] possesses, in addition to the usual derivative term acting on the $p$ 's in the argument of $\Psi$, a contribution giving the spin $s$ :

$$
M=-i \mathbf{p} \times \frac{\partial}{\partial \mathbf{p}}+s
$$

The possibility of adding this arbitrary constant $s$ to the rotation generator reflects the obvious and well-known fact that planar rotations commute and that the Abelian rotation algebra allows an addition, viz., an arbitrary spin. What is not so obvious and perhaps not as well known is that the generators of the full non-Abelian Poincaré group can be similarly modified by an additional term proportional to $s$, and the Lie algebra (2.1) remains intact. The addition is as in Eq. (2.19) for the angular momentum; for the boost generators $\mathbf{J}$ it is given by

$$
\Delta \mathbf{J}=s \frac{\mathbf{P}}{\mathbf{P}^{2}}[\boldsymbol{H}(\mathbf{P})-\boldsymbol{H}(0)] .
$$


Here the dependence of the Hamiltonian $H \equiv P^{0}$ on $\mathbf{P}$ is explicitly indicated.

The modifications in (2.19) and (2.20) may also be presented in covariant notation, which puts into evidence a relation to the magnetic monopole. The Poincare algebra (2.1) is not changed if $-s \Delta^{a}(p)$ is added to $J^{a}$, where $\Delta^{a}$ is given in (2.14). In particular, as is explicitly demonstrated by the induced representation constructed above, the Lorentz generators, which solve the helicity condition in (2.2) on the one-particle momentum space, read

$$
J^{a}=-i \epsilon^{a b c} p_{b} \frac{\partial}{\partial p^{c}}-s \Delta^{a}(p)
$$

Moreover, the unitary operator acting on $\Psi$ is $U(\Lambda)=e^{i \omega_{a} J^{a}}$, with the contribution involving derivatives effecting the Lorentz transformation on the argument of $\Psi$, and $-s \Delta^{a}$ giving rise to the one-cocycle $e^{-i s \omega_{\Lambda, p}}$. Evidently the above induced representation indeed satisfies the requirements of $(2.2)$; it is appropriate to the orbit $p^{0}>0$.

The addition in (2.21) is just what comes from a magnetic monopole (in three-dimensional momentum space with Lorentzian signature). But since we are dealing with $\mathrm{SO}(2,1)$ rather than $\mathrm{SO}(3)$, the addition is nonsingular; $(p \cdot \eta+m)$ never vanishes and $s$ need not be quantized. If one adds the "monopole" field $\left(s / 2 m^{3}\right) \epsilon^{a b c} p_{a} d p_{b} \wedge d p_{c}$ to the standard symplectic form $d x^{a} \wedge d p_{a}$, with $p^{2}-m^{2}=0$, one obtains the modified generators. For discussing the point-particle mechanics of an anyon we can thus use any action that leads to this symplectic structure, as has been done by many authors. ${ }^{6,7}$ This does not however yield a manifestly covariant field theory, which is our goal in this paper.

For a manifestly covariant field theory we have to go beyond the on-shell description given by the induced representation. We have to introduce fields transforming linearly as a spin-s representation of the Lorentz group and satisfying a linear differential equation whose solutions provide the desired induced representation. In general this leads to many more field components than the single physical state required by the above analysis. For integer or half-integer $s$, one conventionally uses $2 s+1$ components, while arbitrary-s representations of the Lorentz group require an infinite number of components. Additional considerations are then needed to eliminate all but one component. It is instructive to examine in detail how this works in the familiar situations with spins $\frac{1}{2}$ and 1 .

\section{FAMILIAR EXAMPLES}

\section{A. Spin $\frac{1}{2}$}

The Dirac equation for a spin- $\frac{1}{2}$ particle,

$$
i \gamma^{a} \partial_{a} \psi \pm m \psi=0
$$

may be realized in $2+1$ dimensions with a twocomponent spinor and $2 \times 2 \gamma$ matrices that are multiples of the Pauli matrices: $\gamma^{a}=\left(-\sigma^{3},-i \sigma^{2}, i \sigma^{1}\right)$. Observe that $\gamma^{a} / 2$ satisfy the $\operatorname{SO}(2,1)$ algebra (2.1); calling them $j^{a}$,

$$
j^{a}=\gamma^{a} / 2,
$$

and noting that the translation operators are realized by $i \partial_{a}=p_{a}$, we can write (3.1) as

$$
p \cdot j \psi \pm \frac{1}{2} m \psi=0
$$

or, with $s= \pm \frac{1}{2}$,

$$
p \cdot j \psi+m s \psi=0 \text {. }
$$

We recognize that the Dirac equation is precisely the Pauli-Lubanski condition of (2.2), now applied to a wave function. ${ }^{8}$

The positive-energy solution to (3.1) (we take the lower sign for the mass term) involves only one function $\Psi(p)$,

$$
\psi(p)=\frac{1}{\sqrt{2 m(E+m)}}\left(\begin{array}{c}
p^{x}-i p^{y} \\
E+m
\end{array}\right) \Psi(p),
$$

which provides a one-component spin- $\frac{1}{2}$ representation. This can be explicitly seen as follows. Observe that $\psi(p)$ of (3.4) may be presented as

$$
\psi(p)=B(p)\left[\begin{array}{c}
0 \\
\Psi(p)
\end{array}\right],
$$

where $B(p)$ is the boost $(2.7),(2.9)$ in the representation (3.2):

$$
\begin{aligned}
B(p) & =e^{i \Omega_{a}(p) \gamma^{a} / 2} \\
& =\frac{1}{\sqrt{2 m}}\left[\sqrt{p \cdot \eta+m}+i \frac{\epsilon^{a b c} p_{a} \eta_{b} \gamma_{c}}{\sqrt{p \cdot \eta+m}}\right] .
\end{aligned}
$$

Since the Lorentz generator $J^{a}$ acting on $\psi(p)$ is

$$
J^{a}=-i \epsilon^{a b c} p_{b} \frac{\partial}{\partial p^{c}}+\frac{1}{2} \gamma^{a},
$$

it acts on $\left[\begin{array}{c}0 \\ \Psi(p)\end{array}\right]$ as

$$
\begin{aligned}
& B^{-1}(p) J^{a} B(p) \\
& =B^{-1}(p)\left[\left(-i \epsilon^{a b c} p_{b} \frac{\partial}{\partial p^{c}}+\frac{1}{2} \gamma^{a}\right) B(p)\right] \\
& \quad=i \epsilon^{a b c} p_{b} \frac{\partial}{\partial p^{c}}+B^{-1}(p)\left[-i \epsilon^{a b c} p_{b} \frac{\partial}{\partial p^{c}}+\frac{1}{2} \gamma^{a}\right) B(p) .
\end{aligned}
$$

From (2.18) we see that the last term in (3.7b) is $\frac{1}{2} \Delta^{a} \gamma^{0}$. Consequently, the action of $J^{a}$ on $\Psi(p)$ is by

$$
J^{a}=-i \epsilon^{a b c} p_{b} \frac{\partial}{\partial p^{c}}+\frac{1}{2} \Delta^{a},
$$

in agreement with (2.21) for $s=\frac{1}{2}$.

Note that the mass-shell condition is not separately imposed; it follows from the Dirac equation by iteration.

\section{B. Spin 1}

A more appropriate example for us is the massive gauge theory. A massive spin-1 particle also requires only a one-component field, according to the Poincare 
group analysis. However, a linear representation of the Lorentz group needs three components. We therefore use a three-component field satisfying a subsidiary condition, in addition to the helicity condition, thereby eliminating the unwanted components. We shall see that the subsidiary condition can also be solved in terms of gauge potentials; the theory can then be rewritten as a gauge theory, the subsidiary condition becoming the Bianchi identity. The elimination of the extra field components is, in this version, an expression of gauge freedom.

The minimal example of a massive gauge theory is the topologically massive theory. ${ }^{9}$ Its equations of motion are usually presented in terms of the field strength $F^{a b}$ :

$$
\partial_{b} F^{b a} \pm \frac{m}{2} \epsilon^{a b c} F_{b c}=0 .
$$

For our purposes it is preferable to write this in terms of a dual field strength $F^{a}$, which is a three-component vector. With the definition

$$
F^{a} \equiv \frac{1}{2} \epsilon^{a b c} F_{b c},
$$

(3.8) becomes $^{10}$

$$
\partial_{a} \epsilon^{a b c} F_{c} \pm m F^{b}=0 .
$$

Again setting $i \partial_{a}=p_{a}$ and recalling from (2.8) the spin-1 representation matrices for the generators of $\operatorname{SO}(2,1)$ we see that (3.10) is again the Pauli-Lubanski scalar, acting as a $3 \times 3$ matrix on the three-vector $F^{a}$, with $s= \pm 1$ :

$$
(p \cdot j)_{b}^{a} F^{b}+m s F^{a}=0 .
$$

A transversality condition follows from (3.10) or (3.11):

$$
p_{a} F^{a}=0 .
$$

This gives a subsidiary condition, which reduces the number of physical components, but it is not imposed separately; rather, it follows from the postulated field equation, as does the mass-shell condition by "squaring" (3.10) and using (3.12).

Let us show explicitly how solutions to the threecomponent equation (3.10) give rise to the spin-1, onecomponent representation of the Poincaré group. The solution to (3.10) (with upper sign for the mass) is

$F^{a}(p)=\frac{1}{\sqrt{2}}\left[\left(\begin{array}{l}0 \\ 1 \\ i\end{array}\right)+\frac{p^{x}+i p^{y}}{m(E+m)}\left(\begin{array}{c}E+m \\ p^{x} \\ p^{y}\end{array}\right)\right] \Psi(p)$.

This may also be presented as

$$
F^{a}(p)=B_{b}^{a}(p) N^{b} F_{0}^{c}(p),
$$

where $B(p)$ is the boost (2.7) in the vector representation, viz. (2.10), $N$ is the numerical unitary matrix

$$
N_{c}^{b}=\frac{1}{\sqrt{2}}\left(\begin{array}{ccc}
\sqrt{2} & 0 & 0 \\
0 & 1 & 1 \\
0 & -i & i
\end{array}\right)
$$

and

$$
F_{0}{ }^{a}=\left(\begin{array}{c}
0 \\
0 \\
\Psi(p)
\end{array}\right) .
$$

The Lorentz generator $J^{a}$ involves the matrix action on $F^{a}$ :

$$
\left(J^{a}\right)^{a^{\prime}}{ }_{b^{\prime}}=-i \epsilon^{a b c} p_{b} \frac{\partial}{\partial p_{c}} \delta_{b^{\prime}}^{a^{\prime}}+i \epsilon_{b^{\prime}}^{a^{\prime} a}
$$

Therefore on $F_{0}{ }^{a}$ the action is by

$$
\begin{aligned}
{\left[N^{-1} B^{-1}(p) J^{a} B(p) N\right]_{b^{\prime}}^{a^{\prime}}=} & {\left[N^{-1} B^{-1}(p)\right]_{a^{\prime \prime}}^{a^{\prime}}\left[\left[-i \epsilon^{a b c} p_{b} \frac{\partial}{\partial p^{c}} \delta_{b^{\prime \prime}}^{a^{\prime \prime}}+i \epsilon_{b^{\prime \prime}}^{a^{\prime \prime} a}\right][B(p) N]_{b^{\prime}}^{b^{\prime \prime}}\right] } \\
= & -i \epsilon^{a b c} p_{b} \frac{\partial}{\partial p^{c}} \delta_{b^{\prime}}^{a^{\prime}} \\
& +\left(N^{-1}\right)^{a^{\prime}}{ }_{a^{\prime \prime}}\left[\left[B^{-1}(p)\right]_{a^{\prime \prime \prime}}^{a^{\prime \prime}}\left[-i \epsilon^{a b c} p_{b} \frac{\partial}{\partial p^{c}} \delta_{b^{\prime \prime \prime}}^{a^{\prime \prime \prime}}+i \epsilon^{a^{\prime \prime \prime} a}{ }_{b^{\prime \prime \prime}}\right][B(p)]_{b^{\prime \prime}}^{b^{\prime \prime \prime}}\right](N)^{b^{\prime \prime}}{ }_{b^{\prime}}
\end{aligned}
$$

Use of (2.18) allows replacing the last bracketed expression by $\Delta^{a} i \epsilon^{a^{\prime \prime} 0}{ }_{b^{\prime \prime}}$; when the remaining contractions with $N$ and $N^{-1}$ are performed, this becomes

$$
\left[\begin{array}{lll}
0 & & \\
& 1 & \\
& & -1
\end{array}\right]_{b^{\prime}}^{a^{\prime}}
$$

Therefore the action on $\Psi(p)$ is by

$$
J^{a}=-i \epsilon^{a b c} p_{b} \frac{\partial}{\partial p^{c}}-\Delta^{a}
$$

in agreement with (2.21) for $s=1$.

Let us observe that the subsidiary condition (3.12) may be viewed as a Bianchi identity, which allows introducing a gauge potential: a transverse vector can be written (in topologically trivial space-time) as a curl:

$$
F^{a}=\epsilon^{a b c} \partial_{b} A_{c} .
$$

Now the description possesses the gauge freedom of adding $\partial_{a} \theta$ to $A_{a}$. The emergent gauge principle, although not essential to understanding one-particle states, is needed to introduce interactions with charged particles. In fact, the dual (3.10) or (3.11) of the topologically massive equation (3.8) can be obtained from a local and manifestly gauge-invariant Lagrange density: varying $F^{a}$ in

$$
\mathcal{L}=\frac{1}{2} F^{a} F_{a} \mp \frac{1}{2 m} \epsilon^{a b c} F_{a} \partial_{b} F_{c}
$$

yields (3.8); in contrast with (3.6) which is derived from a 
Lagrangian constructed from gauge potentials and containing the gauge-invariant Chern-Simons term. Nevertheless gauge potentials must be introduced as in (3.17) if charged matter is to be coupled to the gauge field. In topologically nontrivial space-times (which we shall not consider here) implementing (3.17) may encounter an obstruction, which then constrains the charges governing matter-gauge field couplings (but not the mass $m$ ). ${ }^{11}$

\section{THE ANYON EQUATION}

We take the spin-1 gauge theory as a model and construct a theory of anyons where the fields are infinite component vectors carrying a linear (spin- $\lambda \times$ spin-1) representation of the Lorentz group; the spin of the oneparticle states will turn out to be $s=1-\lambda$. We impose the helicity condition, which we call the Pauli-Lubanski equation, and identify further subsidiary conditions that pick out a single physical state from the infinite number of components. In the next section we solve the subsidiary conditions to exhibit the theory as a gauge theory. As in the topologically massive case, the gauge principle is expected to be useful in understanding interactions of anyons.

For arbitrary $\lambda$ our theory is an infinite component generalization of the topologically massive spin-1 model. The field equations reduce to (3.10) when $\lambda$ is chosen zero.

We begin with a description of the relevant representations of $\mathrm{SO}(2,1)$. Concentrating on arbitrary values of $\lambda$, the representations we use are unitary, infinite dimensional and bounded on one side: they are members of the discrete series of $\mathrm{SO}(2,1) .^{12}$ The bound can be either above or below; these are equivalent choices related by a discrete symmetry. [For nonintegral values of $\lambda$, we have, strictly speaking, representations of a suitable covering of $\mathrm{SO}(2,1)$.]

We denote the Lorentz generators for these representations by $K^{a}$ and the states by $|\lambda, n\rangle, n=0,1, \ldots, \infty$. For representations bounded below, the action of the $K^{a}$ 's on states is given by

$$
\begin{aligned}
& K^{0}|\lambda, n\rangle=(\lambda+n)|\lambda, n\rangle, \\
& K^{+}|\lambda, n\rangle=\sqrt{(2 \lambda+n)(n+1)}|\lambda, n+1\rangle, \\
& K^{-}|\lambda, n\rangle=\sqrt{(2 \lambda+n-1) n}|\lambda, n-1\rangle,
\end{aligned}
$$

where $K^{ \pm}=K^{1} \mp i K^{2}$. (The sign reversal in our definition of $( \pm)$ derives from the metric.) The eigenvalue of $K^{0}$ for the lowest-weight state $|\lambda, 0\rangle$, with $K^{-}|\lambda, 0\rangle=0$, is $\lambda$ and characterizes the representation.

For the representation bounded above we have

$$
\begin{aligned}
& K^{0}|\lambda, n\rangle=-(\lambda+n)|\lambda, n\rangle, \\
& K^{+}|\lambda, n\rangle=-\sqrt{(2 \lambda+n-1) n}|\lambda, n-1\rangle, \\
& K^{-}|\lambda, n\rangle=-\sqrt{(2 \lambda+n)(n+1)}|\lambda, n+1\rangle .
\end{aligned}
$$

The representation is here characterized by the $K^{0}$ eigenvalue $-\lambda$ of the highest-weight state $|\lambda, 0\rangle, K^{+}|\lambda, 0\rangle=0$.

For both representations (4.1) and (4.2) the value of the quadratic Casimir $g_{a b} K^{a} K^{b}$ is $\lambda(\lambda-1)$, and the two are related by $K^{a} \rightarrow-K^{a T}$, where $T$ denotes the transpose, $K^{a}$ 's being considered as infinite-dimensional matrices. This is the basic conjugation symmetry of the theory.

There is a well-known and convenient way of characterizing these representations in terms of functions of a complex variable $z .{ }^{13}$ One assigns a "wave function" $\langle z \mid \lambda, n\rangle$ to the state $|\lambda, n\rangle$ by

$\langle z \mid \lambda, n\rangle=\mathcal{N} z^{n} \quad$ (representations bounded below),

$\langle z \mid \lambda, n\rangle=\mathcal{N} z^{-2 \lambda-n}$ (representations bounded above),

where $\mathcal{N}$ is a normalization factor equal to $\sqrt{\Gamma(2 \lambda+n) / \Gamma(n+1) \Gamma(2 \lambda-1)}, \Gamma$ being the gamma function. The monomials (4.3), (4.4) form a basis for holomorphic functions, analytic in the unit disc $D,|z| \leq 1$ in the first case and in $\widetilde{D}$ with $|z| \geq 1$ in the second case (except for a branch cut of strength $2 \lambda$ ). An arbitrary state of the representation is thus a holomorphic function of the type

$$
f(z)=\sum_{n=0}^{\infty} f_{n}\langle z \mid \lambda, n\rangle,
$$

and on such functions $K^{a}$ are realized by differential operators:

$$
K^{0}=z \partial_{z}+\lambda, \quad K^{+}=z^{2} \partial_{z}+2 \lambda z, \quad K^{-}=\partial_{z} .
$$

The inner product of two holomorphic functions $f(z)$ and $g(z)$ is constructed as

$$
\begin{aligned}
& \langle f \mid g\rangle=\frac{1}{2 \pi i} \int_{D} d z^{*} d z \frac{f^{*} g}{\left(1-z^{*} z\right)^{2-2 \lambda}}, \\
& \langle f \mid g\rangle=\frac{1}{2 \pi i} \int_{\widetilde{D}} d z^{*} d z \frac{f^{*} g}{\left(z^{*} z-1\right)^{2-2 \lambda}},
\end{aligned}
$$

with $d z^{*} d z=2 i r d r d \theta$ in radial coordinates. Equation (4.7) applies to representations bounded below and (4.8) to those bounded above. With this inner product the realization (4.6) for $K^{a}$ is Hermitian, and the group representation is unitary, but infinite dimensional.

Although the representations (4.1) and (4.2) can be defined for any $\lambda$, the above inner product requires $\lambda>\frac{1}{2}$. This is necessary so that the potential singularity at $|z|=1$ is integrable. For values $\lambda<\frac{1}{2}$, but $\lambda \neq-N / 2$, $N=0,1,2, \ldots$, one can still use the inner product (4.6) if suitable analytic continuations are made.

Finite-dimensional representations arise when $\lambda$ takes the exceptional values $\lambda=-N / 2$ in the representation (4.1) bounded below. Since $K^{+}\left|-\frac{1}{2} N, N\right\rangle=0$ the representation is also bounded above and we indeed have finite dimensionality. Basis states are still represented by $\mathcal{N} z^{n}$, but now arbitrary states are polynomials in $z$ of degree $N$. It is clear that the inner product (4.7) cannot be used in these cases because of the singularity at $|z|=1$; also the square roots in (4.1) now acquire factors of $i=\sqrt{-1}$. Either way we see that the finite-dimensional representations are not unitary.

The fields of our theory in components are $F_{n}^{a}(x)$, 
$a=0,1,2$ and $n=0,1, \ldots, \infty . K_{n n^{\prime}}^{a}=\left\langle\lambda, n\left|K^{a}\right| \lambda, n^{\prime}\right\rangle$ are infinite-dimensional matrices acting on the $n^{\prime}$ index of $F_{n^{\prime}}^{a^{\prime}}$. The spin-1 matrices for $\mathrm{SO}(2,1)$, viz., $\left(j^{a}\right)_{a^{\prime} a^{\prime \prime}}=i \epsilon_{a^{\prime}}{ }^{a} a^{\prime \prime}$, act on the vector index $a^{\prime \prime}$ of $F_{n^{\prime}}^{a^{\prime \prime}}$.

Alternatively, by use of the functions (4.3) one can collect $F_{n}^{a}$ into a vector $F^{a}(x, z)$, which is a holomorphic function of $z$, well behaved in the unit disc $D,|z| \leq 1$, for representations bounded below, and in $\widetilde{D}$ for representations bounded above. Explicitly,

$$
F^{a}(x, z)=\sum_{n=0}^{\infty} F_{n}^{a}\langle z \mid \lambda, n\rangle .
$$

$K^{a}$ 's act on $F^{a}(x, z)$ as the differential operators in (4.5).

The Pauli-Lubanski scalar $P \cdot J$ is evidently given by $P \cdot(K+j)$ since $J=K+j$ is the total spin contribution to the Lorentz generators. Our proposed equation is thus

$$
P \cdot(K+j)_{a n a^{\prime} n^{\prime}} F_{n^{\prime}}^{a^{\prime}}+m s F_{a n}=0,
$$

where the spin $s$ is taken equal to $1-\lambda$. The index notation is as follows: $P \cdot K_{a n a^{\prime} n^{\prime}}=P \cdot K_{n n^{\prime}} \delta_{a a^{\prime}}$ $P \cdot j_{a n a^{\prime} n^{\prime}}=P \cdot j_{a a^{\prime}} \delta_{n n^{\prime}}$. Henceforth we suppress the infinite-ranging $n$ index.

We must also impose further conditions, which along with (4.10) lead to the mass-shell condition and ensure that only one polarization or component survives out of the threefold infinity of components in $F_{n}^{a}$. To state these conditions, we define the operator

$$
D_{n n^{\prime}}^{a}=\epsilon_{b c}^{a} P^{b} K_{n n^{\prime}}^{c}
$$

and demand that, in addition to (4.10), $F^{a}$ satisfy $^{14}$

$$
\begin{aligned}
& \epsilon^{a b c} D_{b} F_{c}=0, \\
& D_{a} F^{a}=0 .
\end{aligned}
$$

We first demonstrate that these conditions are adequate by solving (4.10) and (4.12). We take plane-wave solutions of the form

$$
F^{a}=F^{a}(p) e^{-i p \cdot x} .
$$

For the amplitudes $F^{a}(p)$, the same set of equations (4.10), (4.12) holds with $P^{a}$ replaced by the momentum eigenvalue $p^{a}$. Taking the scalar product of (4.10) with $p^{a}$ we get $(p \cdot K+m s)(p \cdot F)=0$. The scalar product of (4.10) with $D_{a}$ yields, by use of the algebra of the $K^{a}$,s, $(p \cdot K)(p \cdot F)=0$. These two together require

$$
p \cdot F=0 \text {. }
$$

The transversality condition thus follows from the postulated equations, just as in the vector case.

To solve the subsidiary conditions (4.12), we introduce a triad of unit vectors $\left(e_{0}^{a}, e_{1}^{a}, e_{2}^{a}\right): e_{0}^{a}$ is a timelike vector parallel to $p^{a}$, and $e_{1}^{a}, e_{2}^{a}$ are spacelike and form a basis for vectors orthogonal to $p^{a}$. These satisfy

$$
e_{m}^{a} e_{n}^{b} g_{a b}=g_{m n}, \quad e_{m}^{a} e_{n}^{b} g^{m n}=g^{a b}, \quad e_{b c}^{a} e_{m}^{b} e_{n}^{c}=\epsilon_{m n}{ }^{p} e_{p}^{a},
$$

where the metric tensor $g_{m n}$ governing the ordinal indices $\{m, n, p\}=\{0,1,2\}$ is the same as in space-time: $g_{m n}=\operatorname{diag}(1,-1,-1)$. Explicit expressions can be

$$
e_{0}^{a}=\frac{p^{a}}{\sqrt{p^{2}}}, \quad e_{1}^{a}=\left(0, \epsilon^{i j} \hat{p}^{j}\right), \quad e_{2}^{a}=\frac{1}{\sqrt{p^{2}}}\left(|\mathbf{p}|, \hat{p}^{i} p_{0}\right) .
$$

The combinations $e_{ \pm}^{a}=e_{1}^{a} \mp i e_{2}^{a}$ and $e_{0}^{a}$ are eigenstates of $(p \cdot j)_{a b}=i \sqrt{p^{2}} \epsilon^{a c b} e_{0}^{c}$ :

$$
(p \cdot j)^{a}{ }_{b} e_{ \pm}^{b}= \pm \sqrt{p^{2}} e_{ \pm}^{a}, \quad(p \cdot j)^{a}{ }_{b} e_{0}^{b}=0 .
$$

Equation (4.14) shows that $F^{a}$ has no component along $p^{a}$. We can thus write

$$
F^{a}=F_{+} e_{-}^{a}+F_{-} e_{+}^{a} .
$$

Equations (4.12) can now be simplified to

$$
K_{(+)} F_{-}=0, \quad K_{(-)} F_{+}=0,
$$

where $K_{( \pm)}=K_{a} e_{ \pm}^{a}$ are raising and lowering operators for the eigenvalues of $K_{(0)}=K_{a} e_{0}^{a}$, as may be seen from the commutation rules. For a spin- $\lambda$ representation bounded below, (4.19) can be satisfied if we choose $F_{-}=0$ and if we allow for $F_{+}$one nonvanishing component corresponding to the lowest-weight state of $K_{(0)}$ with $K_{(-)} F_{+}=0$. The nonvanishing amplitude is arbitrary. The Pauli-Lubanski equation (4.10) is satisfied with this component being arbitrary, provided $\sqrt{p^{2}}=m$, or $p^{2}=m^{2}$. (Recall that $s=1-\lambda$.)

We conclude that the subsidiary conditions (4.12) along with the Pauli-Lubanski equation (4.10) do indeed lead to plane-wave solutions with a one-component amplitude, i.e., one polarization, satisfying the mass-shell condition. The solution we have found for representations bounded below corresponds to a positive-energy solution in the language of one-particle quantum mechanics. With our choice of sign for the $m s=m(1-\lambda)$ term in the PauliLubanski equation, there is no negative-energy solution for representations bounded below.

For representations bounded above, Eqs. (4.19) have the solution $F_{+}=0$, with $F_{-}$retaining one nonvanishing component corresponding to the highest-weight state, annihilated by $K_{(+)}$. In this case, the Pauli-Lubanski equation (4.10) can also be satisfied provided $\sqrt{p^{2}}=-m$; in other words, one has a negative-energy solution. The association of the negative-energy solution with the representation bounded above, which is the conjugate of the representation bounded below under the $K^{a} \rightarrow-K^{a T}$ transformation, is as expected. Also, if the sign of the $m s=m(1-\lambda)$ term in (4.10) is changed, the role of these two representations gets exchanged. This property again parallels similar features for spins $\frac{1}{2}$ and 1 that we have discussed.

The nonvanishing amplitude for our solutions corresponds to either the highest- or lowest-weight state of $K_{(0)}$. In the rest frame, $K_{( \pm)}$and $K_{(0)}$ become $K^{ \pm}$and $K^{0}$ as given by (4.1); the nonvanishing amplitude is then $F_{+, 0}\left(\right.$ or $\left.F_{-, 0}\right)$. The amplitude, for arbitrary p, can be written as the Lorentz-boosted version of $F_{+, 0}$ (or $F_{-, 0}$ ). The general solution can thus be written as

$$
F_{n}^{a( \pm)}(p)=B_{n 0}(p) B^{a}{ }_{b}(p) N^{b}{ }_{c} f^{c( \pm)}(p),
$$


where

$$
f^{c(+)}(p)=\left[\begin{array}{c}
0 \\
0 \\
\Psi^{(+)}(p)
\end{array}\right), \quad f^{c(-)}(p)=\left[\begin{array}{c}
0 \\
\Psi^{(-)}(p) \\
0
\end{array}\right] ;
$$

$B_{n n^{\prime}}(p)$ and $B_{b}^{a}(p)$ are the spin- $\lambda$ and spin-1 representations of the boost transformation, respectively, while $N^{b}{ }_{c}$ is the numerical matrix (3.15). The $\Psi^{( \pm)}(p)$ are arbitrary functions, providing one-component representations for the Poincaré group with positive $(+)$ and negative $(-)$ energy. From the property (2.18), we see that the Lorentz generators on $\Psi^{( \pm)}(p)$ have the expected addition $-s \Delta^{a}$ as in (2.21). The solutions of our covariant equations (4.10) and (4.12) do indeed reproduce the induced representation analysis of $\mathrm{Sec}$. II.

Finally, we note that, when $\lambda=0$, the action of the $K^{a}$ 's is trivial and the equations become, as promised, those of the topologically massive spin-1 theory. Other equations, making use of nonunitary, finite-dimensional representations, are obtained by choosing $\lambda=-\frac{1}{2} N$, as explained above. Thus for $\lambda=-\frac{1}{2}, N=1$, the function $f(z)$ of $(4.5)$ becomes $f(z)=f_{1}+f_{2} z$, and $\Psi_{\alpha}=\left(f_{1}, f_{2}\right)$ transforms as a two-spinor. Because our theory makes use of vector-valued states, we need to construct (4.9), which now corresponds to $F^{a}(x, z)=F_{1}^{a}(x)+F_{2}^{a}(x) z$, and the two vectors $F_{1,2}^{a}$ combine into the spin-vector $\Psi_{\alpha}^{0}$, appropriate to a $s=1-\lambda=\frac{3}{2}$ equation.

\section{GAUGE-THEORETIC FORMULATION}

We now turn to the interpretation of our anyon theory given by Eqs. (4.10), (4.12) as a gauge theory. A slight modification of the basic equations is useful in setting up the gauge-theoretic version.

We extend the vectorial quantities $D^{a}$ and $F^{a}$ to a fourth component, labeled by the index 3 , which can be equivalently upper or lower. $D^{3}=D_{3}$ is defined by

$$
D_{3}=P \cdot K \text {. }
$$

The new field $F^{3}=F_{3}$ is taken to transform as a spin- $\lambda$ representation of the Lorentz group; just like $F^{a}$, it is an infinite-component vector. The field equations (4.10) and subsidiary conditions (4.12) are altered to read

$$
\begin{aligned}
& \left(D_{3}+p \cdot j+m s\right)_{a b} F^{b}-D_{a} F^{3}=0, \\
& \epsilon^{a b c} D_{b} F_{c}-i P^{a} F^{3}=0, \\
& D_{a} F^{a}+D_{3} F^{3}=0 .
\end{aligned}
$$

Equations (5.2) are in fact equivalent to (4.10), (4.12), because $F^{3}$ is just an auxiliary field, whose contribution, we shall presently show, vanishes as a consequence of (5.2). But first, we rewrite Eqs. (5.2) in a more compact and useful four-dimensional notation.

We combine $F^{a}, F^{3}$ as $F^{\mu}=\left(F^{a}, F^{3}\right)$ and $D^{a}, D^{3}$ as $D^{\mu}=\left(D^{a}, D^{3}\right), \mu=0,1,2,3$. The metric for four-vectors and tensors is $g_{\mu \nu}=\operatorname{diag}(1,-1,-1,1)$. We use the completely antisymmetric tensor $\epsilon_{\mu v \alpha \beta}$ with $\epsilon_{a b c 3}=\epsilon_{a b c}$. Also, we introduce $P^{\mu}=\left(P^{a}, 0\right) ; P^{3}$ is taken to be zero for the time being, but this will be relaxed shortly. In this fourdimensional notation, Eqs. (5.2a) and (5.2b) can be com- bined into

$$
\left(D_{\mu}+s Q_{\mu}\right) F_{v}-\left(D_{\mu}+s Q_{v}\right) F_{\mu}=i \epsilon_{\mu v \alpha \beta} P^{\alpha} F^{\beta},
$$

where $Q_{\mu}=(0,0,0, m)$. Equation $(5.2 \mathrm{c})$ evidently becomes

$$
D_{\mu} F^{\mu}=0
$$

We now show that Eqs. (5.3) and (5.4) [or (5.2)] are equivalent to (4.10) and (4.12). From our definitions we have $P_{\mu} D^{\mu}=0$ and $P_{\mu} Q^{\mu}=0$. Further, the algebra of $K^{a}$ 's leads to the following commutation rules from the $D_{\mu}$ 's:

$$
\left[D_{\mu}, D_{v}\right]=i \epsilon_{\mu \nu \alpha \beta} P^{\alpha} D^{\beta} \text {. }
$$

Consider now the (four-dimensional) dual version of (5.3), which reads

$$
\epsilon_{\alpha \beta \mu v}\left(D^{\mu}+s Q^{\mu}\right) F^{v}=i\left(P_{\alpha} F_{\beta}-P_{\beta} F_{\alpha}\right) .
$$

Upon acting on this equation by $D^{\beta}$ and simplifying the first term by use of the commutation rules (5.5), we get, with the help of (5.4),

$$
i D_{\alpha}(P \cdot F)+s \epsilon_{\alpha \mu \nu \omega} Q^{\mu} D^{v} F^{\omega}=0 .
$$

This equation further gives

$$
(Q \cdot D)(P \cdot F)=0 \text {. }
$$

The scalar product of (5.3) with $P^{\mu}$ gives $\left(D_{v}+s Q_{v}\right)(P \cdot F)=0$, which also implies

$$
\left(Q \cdot D+s m^{2}\right)(P \cdot F)=0 \text {. }
$$

Equations (5.8), (5.9) together require

$$
P \cdot F=0,
$$

which is of course the constraint (4.14) once again. Since $P \cdot F$ vanishes, (5.7) leaves

$$
\epsilon_{\alpha \mu v \omega} Q^{\mu} D^{v} F^{\omega}=0 \text {. }
$$

The scalar product of (5.6) with $Q^{\beta}$ gives, upon use of the above equation and $Q \cdot P=0$,

$$
P_{\mu}(Q \cdot F)=0 \text {. }
$$

Thus $Q \cdot F$, which is essentially $F^{3}$, is a constant and $D_{\mu}(Q \cdot F)$ is also zero. We see that Eqs. (5.2), or equivalently (5.3), (5.4), reduce to (4.10), (4.12); $Q \cdot F$ or $F^{3}$ is an auxiliary field whose contribution vanishes for consistency and Eqs. (5.3), (5.4) define our theory of anyons.

Thus far the four-vectors and tensors we have introduced are merely a notational improvement. However, notice that we can write $D_{\mu}$ as

$$
D_{\mu}=P^{\mu} K_{\mu v}
$$

where $K_{\mu \nu}$ is antisymmetric and $K_{3 a}=K_{a}, K_{a b}=\epsilon_{a b c} K^{c}$. The tensor $K_{\mu v}$ is anti-self-dual in the four-dimensional sense. Its components are generators for one of the $\mathrm{SO}(2,1)$ 's in the decomposition $\mathrm{SO}(2,2) \sim \mathrm{SO}(2,1)$ $\times \mathrm{SO}(2,1), \operatorname{SO}(2,2)$ being the four-dimensional Lorentz group with our metric $g_{\mu v}=\operatorname{diag}(1,-1,-1,1)$. We can also relax the conditions $P^{3}=0$ and $Q_{\mu}=(0,0,0, m)$, re- 
placing them by the four-dimensionally invariant requirements $Q^{2}=m^{2}, P \cdot Q=0$.

Consider Eqs. (5.3), (5.4) with $P^{\mu}, Q^{\mu}$ arbitrary except for $Q^{2}=m^{2}$, and $Q \cdot P=0$. One can reduce (5.3), (5.4) with these constraints by choosing a representative $Q^{\mu}$, say $(0,0,0, m)$, whereupon we recover (5.2). From the definition (5.13) for $D_{\mu}$, one can also check that the commutation rules $(5.5)$, which are necessary to show that both $P \cdot F$ and $P_{\mu}(Q \cdot F)$ vanish, are true even if $P^{3} \neq 0$. We can thus define our theory as the reduction to the $\operatorname{SO}(2,2)$ orbit $Q^{2}=m^{2}, Q \cdot P=0$ of Eqs. (5.3), (5.4) which are defined for arbitrary $P^{\mu}, Q^{\mu}$.

We now turn to the gauge-theoretic interpretation of (5.3), (5.4). In looking for such an interpretation, one of the equations must be regarded as the field equation, while the other is to be regarded as the Bianchi identity, which leads to the introduction of potentials. In analogy to the topologically massive theory, we shall retain Eq. (5.3) as the field equation and take (5.4) as the Bianchi identity. Since the dual of $F^{\mu}$ with $\epsilon_{\mu v \alpha \beta}$ is a three-index antisymmetric tensor, we may expect the potentials to be a two-index antisymmetric tensor $A_{\alpha \beta}$. Indeed, one can easily check that a solution to (5.4) is given by

$$
F_{\mu}=D^{v} A_{\mu v}+i P^{v} \tilde{A}_{\mu v},
$$

where $\tilde{A}_{\mu \nu}$ is dual to $A^{\alpha \beta}$ :

$$
\widetilde{A}_{\mu v}=\frac{1}{2} \epsilon_{\mu v \alpha \beta} A^{\alpha \beta} \text {. }
$$

The field strengths $F_{\mu}$ are invariant under the gauge transformation

$$
A_{\mu v} \rightarrow A_{\mu \nu}^{\prime}=A_{\mu v}+\epsilon_{\mu v \alpha \beta} D^{\alpha} \theta^{\beta}-i\left(P_{\mu} \theta_{v}-P_{v} \theta_{\mu}\right)
$$

where the gauge parameters $\left(\theta_{a}, \theta_{3}\right) \equiv \theta_{\mu}$ transform as spin- $\lambda \times$ spin- 1 and spin- $\lambda$ representations, respectively. Equations (5.3) and (5.14) define the gauge theory of anyons. It should be noted that the gauge potentials and parameters are in general complex since the spin- $\lambda$ representations are complex. The gauge symmetry is also evidently Abelian.

The potentials $A_{\alpha \beta}$ can be decomposed as two threevector potentials $\left(A_{a}, B_{a}\right)$ via $A_{3 a}=A_{a}, A_{a b}=\epsilon_{a b c} B^{c}$, each of them transforming as the spin- $\lambda \times$ spin-1 representation of $\mathrm{SO}(2,1)$. When $\lambda$ and correspondingly $K^{a}$ are chosen to be zero, the potential $B_{a}$ and gauge parameters $\theta_{a}$ can be consistently set to zero. The definition of the field strength (5.14) and the gauge transformations (5.16) become those of the topologically massive theory, $A_{a}$ being the vector potential and $\theta_{3}$ the gauge function.

We close this discussion by noting that a suitable gauge-fixing condition on the potentials is given by $A_{a b}=\epsilon_{a b c} B^{c}=0$. The gauge-fixed equations of motion can be solved easily to show that $A_{a}$ has one polarization, as expected.

We now rewrite the gauge theoretic equations in another way that also clarifies why $(5.14)$ is a solution to (5.4). Given a closed algebra such as (5.15), we can define an associated Becchi-Rouet-Stora-Tyutin- (BRST-) type operator $Q$ and a conjugate operator $\bar{Q}$ in a standard way. ${ }^{15}$ Towards this end, one introduces ghosts $c_{\mu}$ and antighosts $\bar{c}^{\mu}$ with the anticommutation rules

$$
\left\{c_{\mu}, c_{v}\right\}=0, \quad\left\{\bar{c}^{\mu}, \bar{c}^{v}\right\}=0, \quad\left\{c_{\mu}, \bar{c}^{v}\right\}=\delta_{\mu}^{v} .
$$

The BRST operators are then

$$
\begin{aligned}
& Q=c^{\mu}\left(D_{\mu}+\frac{i}{2} \epsilon_{\mu v \alpha \beta} P^{\beta} c^{\nu} \bar{c}^{\alpha}\right), \\
& \bar{Q}=\bar{c}^{\mu}\left(D_{\mu}+\frac{i}{2} \epsilon_{\mu v \alpha \beta} P^{\beta} \bar{c}^{v} c^{\alpha}\right) .
\end{aligned}
$$

It is easily checked that $Q^{2}=0=\bar{Q}^{2}$.

The ghost fields $c_{\mu}, \bar{c}^{\mu}$ are assigned ghost numbers 1 and -1 , respectively; correspondingly $Q$ and $\bar{Q}$ are operators of ghost numbers 1 and -1 , respectively. We can now introduce functions of the type $G(x), \bar{c}^{\mu} G_{\mu}(x)$, $\frac{1}{2} \bar{c}^{\mu} \bar{c}^{v} G_{\mu v}(x), \ldots$ of ghost numbers $0,-1,-2, \ldots$, which are essentially exterior forms relevant to the $D_{\mu}$ 's and the algebra $(5.5) ;^{16}$ i.e., they stand in the same relationship to $D_{\mu}$ 's and (5.5) as ordinary differential forms to $\partial_{\mu}$ and its algebra $\left[\partial_{\mu}, \partial_{v}\right]=0$. For $F^{\mu}$ we introduce the three-form

$$
\mathscr{F}=\frac{1}{3 !} \epsilon_{\mu \nu \alpha \beta} \bar{c}^{v} \bar{c}^{\alpha} \bar{c}^{\beta} F^{\mu}
$$

In terms of this, Eqs. (5.3) and (5.4) become

$$
\begin{aligned}
& Q \mathscr{F}+s\left(Q^{\mu} c_{\mu}\right) \mathscr{F}=0, \\
& \bar{Q} \mathfrak{F}=0 .
\end{aligned}
$$

The Bianchi identify (5.21) is evidently solved by $\mathscr{F}=\bar{Q} \mathcal{A}$, where $\mathcal{A}=\frac{1}{2} \bar{c}^{\mu} \bar{c}^{v} \widetilde{A}_{\mu v}$ is a form of ghost number -2 . This is in fact the easiest way to see that (5.14) is a solution to (5.4). The gauge transformations are $\mathcal{A} \rightarrow \mathcal{A}+\bar{Q} \Theta$, where $\Theta=\bar{c}^{\mu} \theta_{\mu}$.

$Q$ and $\bar{Q}$ are associated with the $D_{\mu}$ 's; there is of course an operator $Q$ associated with the gauge symmetry (5.16) and its gauge fixing. From $\mathcal{A} \rightarrow \mathcal{A}+\bar{Q} \Theta$ we see that there is a second-generation gauge symmetry $\Theta \rightarrow \Theta+\bar{Q} f$, where $f$ has zero ghost number. In other words, the gauge symmetry (5.16) is reducible in the Batalin-Fradkin-Vilkovisky sense and $Q$ will involve second-generation ghosts and antighosts. ${ }^{17}$

\section{ACTION FORMULATION}

We shall now construct an action from which our field equations can be derived as the variational equations. The equations of motion, as they are, do not admit an action formulation. The equations can be written as

$$
\int \delta A_{\mu \nu}^{*} \epsilon^{\mu v}(A)=0
$$

for arbitrary $\delta A_{\mu \nu}^{*}$ (asterisk denotes complex conjugation); $\epsilon_{\mu v}(A)$ is the equation of motion in (5.3). In order to have an action formulation, we must be able to write $\int \delta A_{\mu \nu}^{*} \epsilon^{\mu \nu}=\delta S$; it can be easily checked that the integrability conditions for this are not satisfied by the $\left(Q_{\mu} F_{v}-Q_{v} F_{\mu}\right)$ term in the equation of motion. We must therefore modify the equation somewhat to obtain an action. 
A suitably modified set of equations is

$$
\begin{aligned}
& \left(D_{3}+P \cdot j\right)_{a b} F^{b}+m s F_{a}-D_{a} F_{3}=0, \\
& \epsilon^{a b c} D_{b} F_{c}+m s \int_{y} \Delta^{a b}(x, y)\left(\epsilon_{b c d} D^{c} F^{d}\right)(y)-i P^{a} F^{3}=0,
\end{aligned}
$$

where $\Delta^{a b}$ is the inverse of $\left(D_{3}+P \cdot j\right)_{a b}$, and $F_{\mu}$ is expressed in terms of the potential as in (5.14), so that $(5.2 \mathrm{c})$ is identically true. These modified equations do admit an action, but it is no longer possible to obtain $\epsilon_{a b c} D^{b} F^{c}=0$ from (6.2). We must supplement (6.2) with

$$
\epsilon_{a b c} D^{b} F^{c}=0 \text {. }
$$

The set of equations (6.2), (6.3) can be derived from the action

$$
\begin{gathered}
S=\int d^{3} x\left[F_{\mu}^{*} F^{\mu}+m s \int_{y} F_{a}^{*}(x) \Delta^{a b}(x, y) F_{b}(y)\right. \\
\left.+(D \times F)_{a}^{\dagger}(D \times F)_{b} \sigma^{a b}\right]
\end{gathered}
$$

where $\sigma^{a b}$ is a Hermitian tensor field and
$(D \times F)_{a} \equiv \epsilon_{a b c} D^{b} F^{c}$ (dagger denotes Hermitian conjugation). The variational equation for $\sigma^{a b}$ gives the constraint $(D \times F)_{a}^{\dagger}(D \times F)_{b}=0$, which is equivalent to $(D \times F)_{a}=0$. The other variational equations, with this condition, reproduce the field equations (5.2).

A term linear in $(D \times F)_{a}$ such as $\sigma^{a}(D \times F)_{a}$ would also lead to Eq. (6.3), but would modify Eqs. (6.2) by terms involving $\sigma^{a}$. Requiring the square of $(D \times F)_{a}$ to vanish seems to be the most economical way of obtaining an action formulation of our equations. The technique of requiring the square of a constraint as the most innocuous way of obtaining it is known in another context, viz. the Lagrangian for a chiral boson. ${ }^{18}$ It may be possible, as in the chiral boson case, ${ }^{19}$ that this term can be eliminated in favor of a nonlocal Lagrangian.

The Lagrangian (6.4) has a nonlocal term involving $\Delta^{a b}$, in addition to any possible nonlocality that might arise from elimination of the $\sigma$ term. One can rewrite this term in a local way by introducing more fields. Specifically, we can take as our action

$$
S=\int d^{3} x\left\{F_{\mu}^{*} F^{\mu}-m s\left[H_{a}^{*} F^{a}+F_{a}^{*} H^{a}+H^{* a}\left(D_{3}+P \cdot j\right)_{a b} H^{b}\right]+(D \times H)_{a}^{\dagger}(D \times H)_{b} \sigma^{a b}\right\} .
$$

$H_{a}$ is a gauge-invariant field, the elimination of which via its equation of motion takes us back to the action (6.4). Notice that the action (6.5) involves coupling the gauge field to $H_{a}$, whose dynamics is essentially given by a ChernSimons-like action.

In (6.4) or (6.5), the fundamental fields are the gauge potentials (and $H_{a}, H_{a}^{*}, \sigma^{a b}$ ), the equations of motion being obtained by extremization with respect to these.

In the case of the topologically massive spin-1 theory, there is an alternative action (3.18) for which the (dual) field strengths are considered as the fundamental fields. There is an analogous action for our theory given by

$S_{\mathrm{dual}}=\int d^{3} x\left[F^{* a}\left(D_{3}+P \cdot j+m s\right)_{a b} F^{b}+V^{* a}(D \times F)_{a}+V^{* 3}(D \cdot F)+(D \times F)_{a}^{\dagger} V^{a}+(D \cdot F)^{\dagger} V^{3}+(D \times V)_{a}^{\dagger}(D \times V)_{b} \sigma^{a b}\right]$.

$V^{a}, V^{3}$, their conjugates and $\sigma^{a b}$ are again auxiliary or Lagrange multiplier fields. (This version may have some advantages with regard to quantization, since only gauge-invariant fields are involved.)

Needless to say, (6.5) and (6.6) reduce, respectively, to the potential and (dual) field-strength versions of the action for the topologically massive theory when $\lambda$ is taken to be zero.

In (6.4), (6.5), or (6.6), terms of the type $F_{a}^{*} F^{a}$ involve summation over all components, e.g., $F_{a}^{*} F^{a}$ $=\sum_{n=0}^{\infty} g_{a b} F_{n}^{* a} F_{n}^{b}$. We can consider the fields as holomorphic functions on the unit $\operatorname{disc} D,|z| \leq 1$ (or $\widetilde{D}$, $|z| \geq 1$ ); the action would then involve integration over $D$ (or $\widetilde{D}$ ) with the measures indicated in (4.7), (4.8).

\section{CONCLUDING REMARKS}

We have obtained a relativistic theory of anyons with manifest Lorentz invariance. Moreover, the extra polarizations are eliminated by suitable constraints. The theory can be considered as a gauge theory, the constraints being the Bianchi identities. The solutions of the field equations realize the one-particle states as the appropriate induced representation of the Poincaré group.
The action requires auxiliary fields; the elimination of these, or of the constraints they imply, would lead to nonlocality.

It is unlikely that one can obtain a simple local Lagrangian in view of the following. Even in $2+1$ dimensions, spin has to be an integer or half-integer for local fields. Fields which carry charges associated with gauge symmetries (with accompanying flux integrals at infinity) are typically localizable only in spacelike cones; for such fields fractional spins are admissible. ${ }^{20}$ The method of generating fractional spin by coupling point particles to a Chern-Simons gauge field, with its nontrivial long-range properties, exemplifies this situation. In our case too, the nonlocalities which arise from the elimination of auxiliary fields, or from enforcing the corresponding constraints, are a reflection of this fact.

We can consider our theory to be a field theory defined on a five-dimensional manifold $\widetilde{M}=M \times N$ where $M$ is the three-dimensional space-time and $N$ is either the unit $\operatorname{disc} D$, or $\widetilde{D}$, or the union of both, if we want to treat positive- and negative-energy solutions on an equal footing. The fields are of course holomorphic on $N$. The modes such as $F_{n}^{a}$ for $n=1, \ldots, \infty$ describe field excitations on $N$ and are similar to Kaluza-Klein modes, which 
arise in dimensional reduction from $\widetilde{M}$ to $M$. However, for us, the modes on $N$ are all gauge degrees of freedom. We may thus think of our theory as a five-dimensional field theory whose (gauge-invariant) dynamics is confined to $M$. From this point of view there is an analogy with anomalous gauge theories where the Wess-Zumino action displays similar features.

We close with a remark about the quantization of the theory. The one-particle states of our theory have arbitrary spin; the phases generated by exchange of particles in a many-particle state must form a representation of the braid group. The anyonic field operators, at least after projecting onto physical polarizations, must therefore have very nontrivial algebraic properties. In particular, the theory has, implicitly, enough information to construct a representation, appropriate to the spin value, of the braid group. Seeing explicitly how this is achieved remains an open problem.

\section{ACKNOWLEDGMENTS}

We thank Michiel Bos and Daniel Freedman for useful discussions. This work was supported in part by funds provided by the U.S. Department of Energy (DOE) under Contract No. DE-AC02-76ER03069.
${ }^{1}$ E. Merzbacher, Am. J. Phys. 30, 237 (1962); J. Leinaas and J. Myrheim, Nuovo Cimento 37, 1 (1977); F. Wilczek, Phys. Rev. Lett. 49, 957 (1982). Early discussions of angular momentum oddities in the plane arose in connection with the Aharonov-Bohm (Ehrenberg-Siday) effect. For a modern review, see F. Wilczek, Fractional Statistics and Anyon Superconductivity (World Scientific, Singapore, 1990).

${ }^{2}$ F. Wilczek and A. Zee, Phys. Rev. Lett. 51, 2250 (1983); M. Bowick, D. Karabali, and L. C. R. Wijewardhana, Nucl. Phys. B271, 417 (1986).

${ }^{3}$ C. Hagen, Ann. Phys. (N.Y.) 157, 342 (1984); Phys. Rev. D 31, 848 (1985); 31, 2135 (1985); D. Arovas, J. Schrieffer, F. Wilczek, and A. Zee, Nucl. Phys. B251, 117 (1985); for a general review, see R. Jackiw, MIT Report No. CTP\#1824, 1989 (unpublished).

${ }^{4}$ J. Hong, Y. Kim, and P. Y. Pac, Phys. Rev. Lett. 64, 2230 (1990); R. Jackiw and E. Weinberg, ibid. 64, 2234 (1990); R. Jackiw and S.-Y. Pi, ibid. 64, 2969 (1990); Phys. Rev. D 42, 3500 (1990); R. Jackiw, K. Lee, and E. Weinberg, ibid. 42, 3488 (1990).

${ }^{5}$ B. Binegar, J. Math. Phys. 23, 1511 (1982).

${ }^{6}$ A. Balachandran, G. Marmo, B. Skagerstam, and A. Stern, Gauge Symmetries and Fibre Bundles (Springer, Berlin, 1983), and references therein.

${ }^{7}$ P. Gerbert, Nucl. Phys. B346, 440 (1990); M. S. Plyushchay, Phys. Lett. B 248, 107 (1990); D. Shon and S. Khlebnikov, Pis'ma Zh. Eksp. Teor. Fiz. 51, 541 (1990) [JETP Lett. 51, 611 (1990)]; S. Forte and T. Jolicoeur, Saclay Report No. SPhT/90-087 (unpublished); see also D. Volkov, D. Sorokin, and V. Tkach, in Problems in Modern Quantum Field Theory, edited by A. Belavin, A. Klimyk, and A. Zamolodchikov (Springer, Berlin, 1989). The approach in the last paper is close to ours, but applies only to spins $\frac{1}{4}$ or $\frac{3}{4}$ and further does not involve a gauge symmetry.

${ }^{8}$ Something similar holds for the Dirac equation in an arbitrary, odd-dimensional space-time. When the dimension is $2 n+1$, a "Pauli-Lubanski scalar" $\mathcal{P}$ may be defined by

$$
\mathcal{P}=N \epsilon_{\mu_{1} \mu_{2}} \cdots \mu_{2 n-1} \mu_{2 n}{ }^{v} P^{v} M^{\mu_{1} \mu_{2}} \cdots M^{\mu_{2 n-1} \mu_{2 n}},
$$

where $N$ is a normalization constant and $M^{\mu \mu^{\prime}}$ generates the $2 d+1$ Lorentz rotations. Taking $M^{\mu \mu^{\prime}}$ proportional to $\left[\gamma^{\mu}, \gamma^{\mu^{\prime}}\right]$, where the $\gamma^{\mu}$ are appropriate "Dirac" matrices, and saturating all but one of the indices in the $\epsilon$ tensor by $\left[\gamma^{\mu}, \gamma^{\mu^{\prime}}\right]$ or equivalently by $\gamma^{\mu} \gamma^{\mu^{\prime}}$ gives rise to the matrix $\epsilon_{\mu_{1} \mu_{2} \cdots \mu_{2 n-1} \mu_{2 n} \nu} \gamma^{\mu_{1}} \gamma^{\mu_{2}} \cdots \gamma^{\mu_{2 n-1}} \gamma^{\mu_{2 n}}$ which in odddimensional space-time is always proportional to $\gamma_{v}$. Hence by adjusting $N, \mathcal{P}$ may be set equal to $P^{v} \gamma_{v}$, so that the kinetic part of the Dirac equation involves solely $P$. This was pointed out to us by D. Freedman.

${ }^{9}$ R. Jackiw and S. Templeton, Phys. Rev. D 23, 2291 (1981); J. Schonfeld, Nucl. Phys. B185, 157 (1981); S. Deser, R. Jackiw, and S. Templeton, Phys. Rev. Lett. 48, 975 (1982); Ann. Phys. (N.Y.) 140, 372 (1982); 185, 406(E) (1988).

${ }^{10}$ S. Deser and R. Jackiw, Phys. Lett. 139B, 371 (1984).

${ }^{11}$ A. Polychronakos, Phys. Lett. B 241, 37 (1990).

${ }^{12}$ See, e.g., B. Wybourne, Classical Groups for Physicists (Wiley, New York, 1974).

${ }^{13}$ See, e.g., I. Gel'fand, M. Graev, and N. Vilenkin, Generalized Functions (Academic, New York, 1966), Vol. 5; S. Lang, $S L_{2} R$ (Springer, Berlin, 1985).

${ }^{14}$ In an earlier, brief account (R. Jackiw and V. Nair, MIT Report No. CTP\#1902, to be published by World Scientific in proceedings of the Sakita Fest) the theory was presented with the field equation $(P \cdot K+m s) F^{a}=0$ and the subsidiary conditions (4.12). While this is more economical than (4.10), it is not possible to derive the transversality condition (4.14), which must therefore be postulated as an additional constraint.

${ }^{15}$ See, e.g., M. Henneaux, Phys. Rep. 126, 1 (1985).

${ }^{16}$ Strictly speaking, the quantities $\left(i \epsilon_{\mu \nu \alpha \beta} P^{\alpha}\right)$ in (5.5) are not structure constants since the derivative operator $P^{\alpha}$ is involved. However, $P^{\alpha}$ commutes with $D_{\mu}$ and can be treated as constant for the algebra of the $D_{\mu}$ 's and the construction of $Q$ and $\bar{Q}$.

${ }^{17}$ I. Batalin and E. Fradkin, Phys. Lett. 122B, 157 (1983); 128B, 303 (1983); I. Batalin and G. Vilkovisky, ibid. 69B, 309 (1977); 102B, 27 (1981).

${ }^{18}$ W. Siegel, Nucl. Phys. B238, 307 (1984).

${ }^{19}$ R. Floreanini and R. Jackiw, Phys. Rev. Lett. 59, 1873 (1987).

${ }^{20}$ D. Buchholz and K. Fredenhagen, Commun. Math. Phys. 84, 1 (1982), J. Fröhlich and P. Marchetti, ibid. 121, 177 (1989). 